\title{
Automated patient and medication payment method for clinical trials
}

This article was published in the following Dove Press journal:

Open Access Journal of Clinical Trials

25 January 2013

Number of times this article has been viewed

\author{
Barbara P Yawn' \\ Suzanne Madison' \\ Susan Bertram' \\ Wilson D Pace ${ }^{2}$ \\ Anne Fuhlbrigge ${ }^{3}$ \\ Elliot Israel ${ }^{3}$ \\ Dawn Littlefield' \\ Margary Kurland' \\ Michael E Wechsler ${ }^{4}$ \\ 'Olmsted Medical Center, \\ Department of Research, Rochester, \\ MN, ${ }^{2}$ UCDHSC, Department of \\ Family Medicine, University of \\ Colorado Health Science Centre, \\ Aurora, CO, 'Brigham and Women's \\ Hospital, Pulmonary and Critical \\ Care Division, Boston, MA, ${ }^{4}$ National \\ Jewish Medical Center, Division \\ of Pulmonology, Denver, CO, USA
}

Correspondence: Barbara P Yawn Olmsted Medical Center, 210 Ninth St SE, Rochester, MN 55904, USA

$\mathrm{Tel}+\mathrm{I} 5072872758$

Fax + I 5072872722

Email byawn@olmmed.org
Background: Published reports and studies related to patient compensation for clinical trials focus primarily on the ethical issues related to appropriate amounts to reimburse for patient's time and risk burden. Little has been published regarding the method of payment for patient participation. As clinical trials move into widely dispersed community practices and more complex designs, the method of payment also becomes more complex. Here we review the decision process and payment method selected for a primary care-based randomized clinical trial of asthma management in Black Americans.

Methods: The method selected is a credit card system designed specifically for clinical trials that allows both fixed and variable real-time payments. We operationalized the study design by providing each patient with two cards, one for reimbursement for study visits and one for payment of medication costs directly to the pharmacies.

Results: Of the 1015 patients enrolled, only two refused use of the ClinCard, requesting cash payments for visits and only rarely a weekend or fill-in pharmacist refused to use the card system for payment directly to the pharmacy. Overall, the system has been well accepted by patients and local study teams. The ClinCard administrative system facilitates the fiscal accounting and medication adherence record-keeping by the central teams. Monthly fees are modest, and all 12 study institutional review boards approved use of the system without concern for patient confidentiality after reviewing all regulatory documents provided by ClinCard.

Conclusion: This system works well for studies that recruit patients from widely dispersed practices and for studies that require flexibility in the amount of payments required, eg, the cost of eight different study medications across varying insurance and pharmacy systems.

Keywords: clinical trial payment, clinical trials, ClinCard

\section{Introduction}

Most clinical trials involve payments to patients for time and burden related to study visits and participation. The ethical issues related to paying patients to participate in research activities have been widely researched and discussed. ${ }^{1-4}$ However, the logistical challenges and potential solutions of making these payments have received little attention. Research participation payments should be easy for the patient to receive and use, as well as cost-effective for the research team to administer and track. Because legal and accounting requirements vary by study, institutional review board, and funding agency, any payment must support fiscal compliance audits and notify the research fiscal agent when patient payments exceed the amount required for federal tax reporting.

The aim of this report is to describe the process of choosing a payment system for a complex comparative effectiveness trial ("the study") currently in progress in 
22 community and academic practices spread across the US. We summarize the benefits and barriers of several options for payment systems evaluated and outline our final selection and the functioning of that system.

BELT (Black Asthmatics and Exacerbations on Longacting Beta Agonists versus Tiotropium) is a randomized clinical trial comparing the effectiveness of a long-acting anticholinergic (tiotropium) plus inhaled corticosteroids versus a long-acting beta-agonist plus inhaled corticosteroids for the prevention of asthma exacerbations. The study is funded by American Recovery and Reinvestment Act (ARRA) funds administered through the Agency for HealthCare Research and Quality.

Enrolled patients must be self-reported Black, 18-75 years of age, report $<10$ pack-years of tobacco use, and have either uncontrolled asthma (by Asthma Control Questionnaire assessment $)^{5}$ while on moderate to high doses of inhaled corticosteroids or currently use a combination of an inhaled corticosteroid plus a long-acting beta-agonist. The study requires five in-person visits to the local study site over the 18 months of enrollment and completion of monthly questionnaires regarding asthma status. Patients are paid fixed amounts for each visit and the return of each monthly questionnaire. In addition, the patients are provided with prescriptions for the study drugs which they obtain at a local pharmacy of their choice. The study pays for medication copays, deductibles, or full cost payment depending on the patient's insurance type. Medication payments vary widely from $\$ 2.00$ copays to over $\$ 500.00$ per month for full cost payments.

\section{Identified options for patient reimbursement}

In many clinical trials, patients are paid for study visits by cash, check, or gift cards. The local practices in our study were unwilling or unable to maintain sufficient cash on hand or rapidly generate checks to pay subjects the $\$ 25-\$ 50$ promised per visit. Furthermore, keeping the required fiscal compliance records of these transactions was deemed highly burdensome by many sites. The central site considered mailing cash, checks, or preloaded gift cards to patients after each study visit, but patients asked for immediate payment and experience from previous studies showed that mailings can result in up to $10 \%$ "lost" cash payments and checks, resulting in significant burden and expense to replace these lost payments.

The variable amounts of medication costs precluded the use of prewritten checks and preloaded gift cards.
Providing large payments to patients rather than paying the pharmacy directly could result in alternative uses of the funds. Conversely, asking patients to self-pay for the medications and then return receipts to the central study site for reimbursement was considered untenable because $25 \%$ of the patients enrolled in our study came from practices serving low-income and uninsured communities. Therefore, a search was undertaken for an alternative payment method, especially for medication payments. We contacted pharmacies in each of the local regions, asking them to dispense medications to the patient and then bill the central site weekly, bi-weekly, or monthly. Only two small locally owned pharmacies were willing to do so. These pharmacies provided medication for fewer than 40 patients each. Other pharmacies stated that they could not afford to carry a large balance on "their books" for 4-6 weeks. National or regional chain pharmacies refused to even discuss this type of delayed payment. Having exhausted the payment systems known to the investigators, the research team explored other options reported in the medical literature or on the Internet.

\section{Materials and methods}

PubMed searches identified no studies or reports that described clinical trial payment systems or methods. An Internet search revealed only one company that specialized in patient payments for clinical trials. ${ }^{6}$ Most systems listed appeared to provide payments to practice study sites and were geared primarily to pharmaceutical companies. ${ }^{7-10}$ Greenphire developed study-specific credit/debit cards called ClinCards that were used to transfer funds rapidly to study sites or study patients. Cards use the MasterCard ${ }^{\circledR}$ company platform and can be used as either a credit or debit card. Card(s) specific for each study are codesigned with the study personnel using study colors, study logo, and any study specific messages that can reasonably fit on the card. Cards are batched to have sequential numbers and all are imprinted with "valuable customer" rather than the patient's name, allowing mass production of the $\operatorname{cards}^{6}$ (Figures 1 and 2).

\section{Patient registration}

Before funds can be uploaded to any card, the card holder (the research patient) must be registered. Patient registration data is entered onto the ClinCard secure website including patient name, address, phone number, and date of birth. During data entry, the system confirms that each entered address is a legitimate one and provides notification when an address is a multifamily dwelling requiring an apartment or condominium number. The registration database can capture 


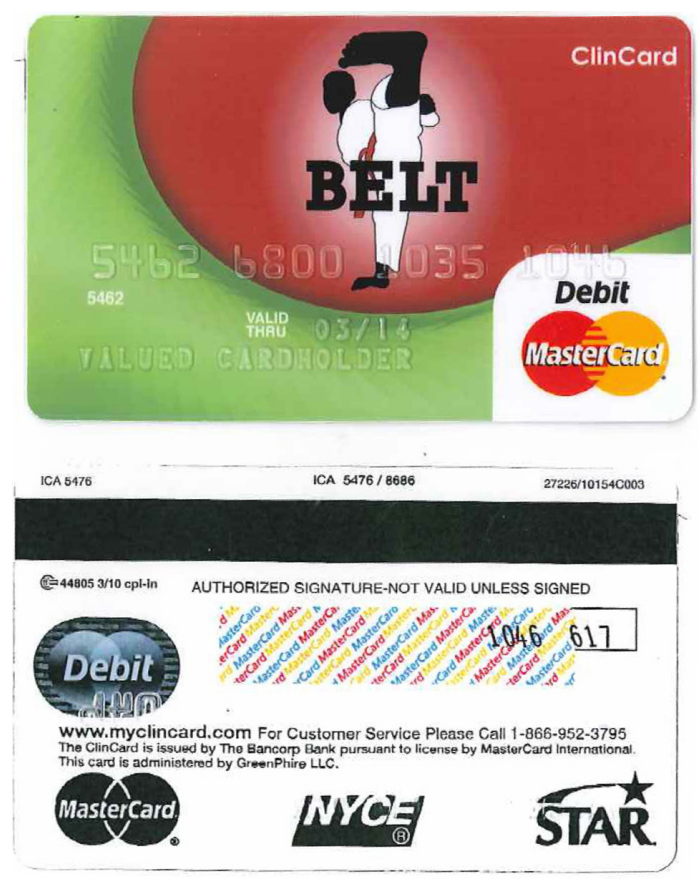

Figure I Stipend card.

home and cell phone numbers as well as an email address which were provided on a volunteer basis by enrolled patients to allow automatic reminders for next visits, if desired by the patient. Cards have unique numbers that are assigned to individual patients. This card number then becomes the basis for patient tracking across the ClinCard system.

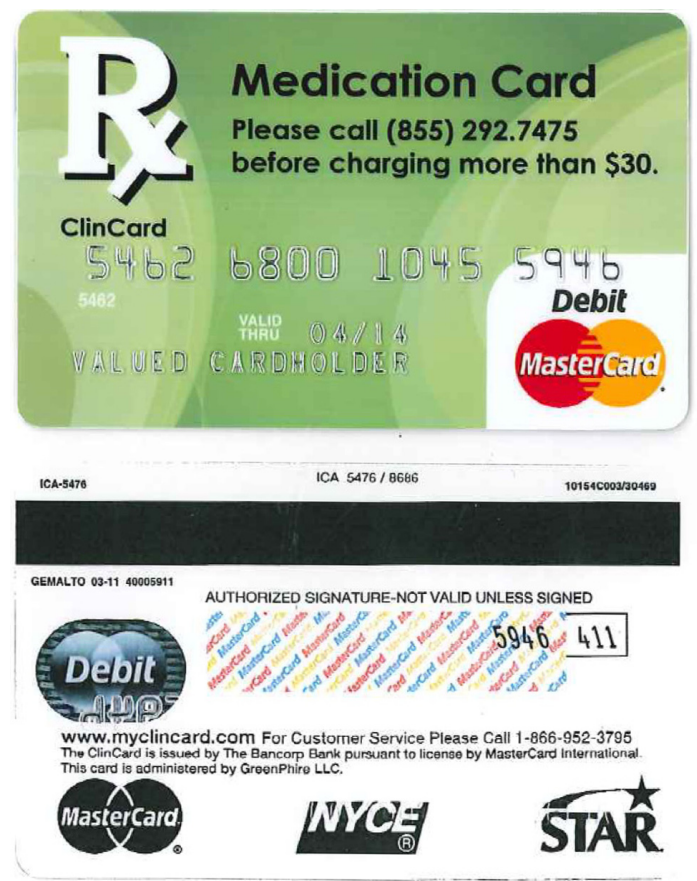

Figure 2 Rx card.

\section{ClinCard administrative systems}

Study staff found the ClinCard web-based administration system to be user-friendly. A limited number of individuals at the central study site were issued passwords to access the system for initial patient data entry and loading funds for study visits or pharmacy calls. Up to five different studies or card systems can be on a single web-based ClinCard administrative system. We currently use it for two large multicentered studies.

The BELT study provides each patient with two cards. A "stipend" card is used to reimburse for study visits and an "Rx" card for pharmacy payments. To place a payment on either card, the patient's account can be identified by their study ID numbers, ClinCard number, or their first or last names or their initials. The multiple methods have been helpful in allowing us to respond equally rapidly to local sites that generally provide patient ID numbers and to pharmacies that use names. For patients with the same name, searching by the patient ID number or the last four digits of the card can distinguish between those patients. For loading funds onto the stipend card, a drop down menu makes the standard payments for each required study visit easy to select, avoiding hand entry and data entry errors. When entering payment amounts onto the Rx card, a comment box is included. This box is used to list the specific drug name, dose and individual costs. The online system has an activity/audit history for each patient that shows dates, amounts, and all notations made for previous payments to that specific card. This feature has been used to track our adherence metrics (Figure 3). The ClinCard system has special features that can be tailored for each study. For example, the web system has a place to enter the date of the next visit which triggers a visit reminder sent to the patient by text or email for patients who have signed consent for this and provided a cell phone number or email address. The system can develop and send a 1099 tax form for all individuals whose payments are over the state or federal limit that requires Internal Revenue Service reporting.

\section{ClinCard costs}

The costs for the credit card system include specific fees for card design, card printing, payment to upload a card with funds, year-end individual 1099 preparation, and a monthly minimum charge. The fees are modest and less than those charged for many gift cards. The fees and startup charges were also less than the costs of writing checks and mailing them for individual payments, and avoided the extra costs of resending payments lost in the mail. Uploads from the study to the ClinCard system are done by electronic bank fund transfers without charge in amounts of $\$ 500$ or more during 


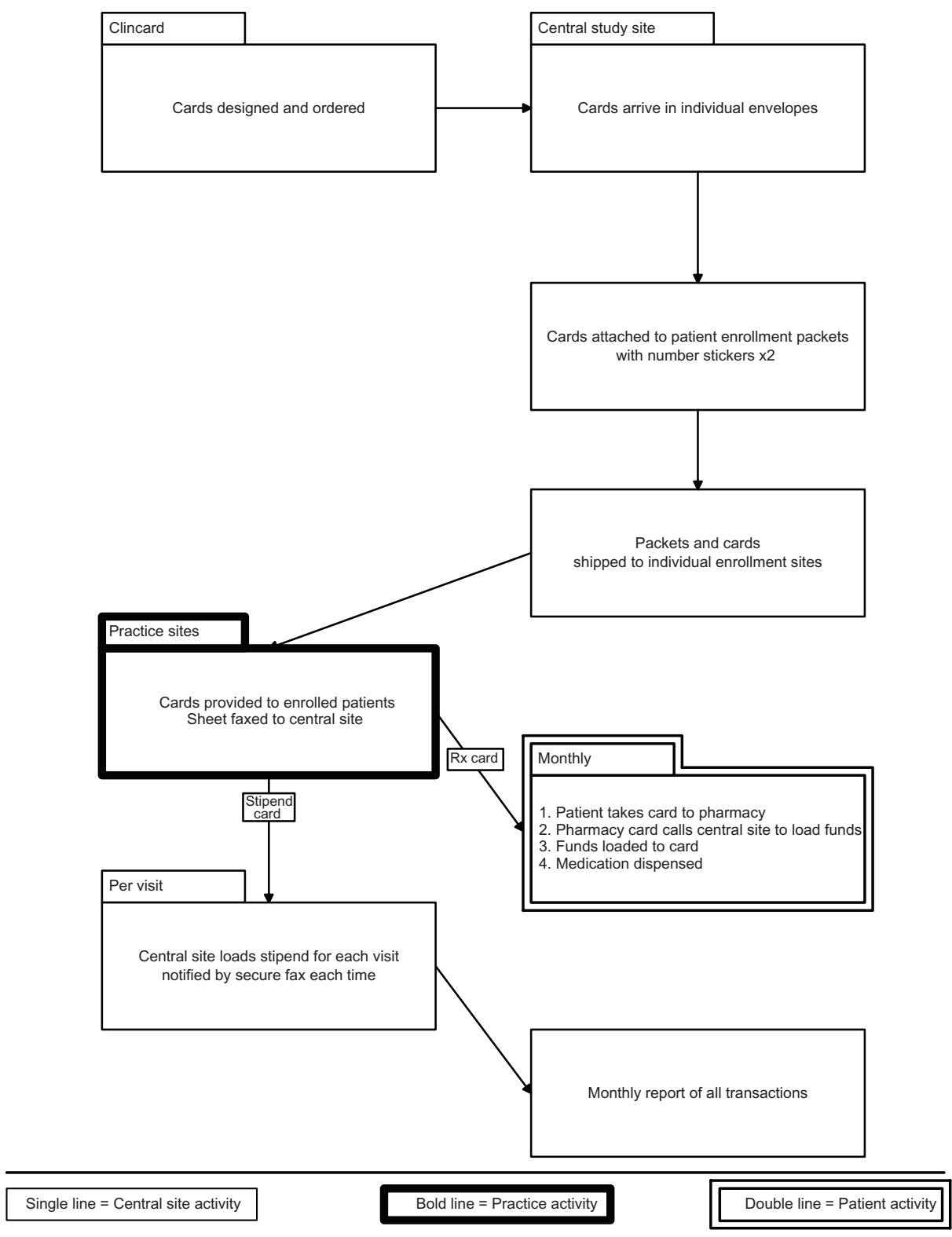

Figure 3 Use of card payment system.

usual bank business hours. Replacement cards can be ordered by the patient online. Alternatively, we ordered extra cards and often have the patient return to their enrollment site for a replacement card. Replacement fees of $\$ 7.00$ are charged by the company. The study team has chosen to replace the card once without charge and then charge the usual replacement fee for the second lost card.

\section{Materials and methods} Study-specific modifications of ClinCard system

The BELT stipend and Rx cards were designed to facilitate the patient's ability to distinguish between their stipend card, the pharmacy payment card, and any other cards they may use. Both cards include the BELT study logo which does not mention asthma and thereby protects patient confidentiality. The pharmacy card has a large Rx on the front and a statement directed to the pharmacist, asking them to call a toll free number prior to charging any amount to the card (Figures 1 and 2). The Rx system was designed to remove the patient from the payment interaction.

The central site developed patient enrollment packets that included both a stipend and Rx card. Each packet was labeled with the patient's study ID and the numbers of the stipend and Rx card. Included with the cards are the details of card use, including a card support number to allow the 
patient to interact with the MasterCard company directly to check balances or to set a PIN. We discouraged use of the PIN because PINs are often forgotten and could delay use of card funds, especially for the Rx card.

Maintaining accurate information on the card numbers given to specific patients is critical, so the central team designed a set of duplicate stickers for the front of each patient enrollment envelope. The duplicate stickers are placed on the front of the site's patient enrollment folder and on the enrollment data sheet faxed to the central site (Figure 3). This assured that records at the practice site and the central site included the patient assigned stipend and Rx card numbers. The information is faxed to the central site and entered by hand into the ClinCard system. As is true for any hand entered data, on occasion data entry errors occurred. Initially it was necessary to enter the patient demographic data twice, once for the stipend card and once for the Rx card making the likelihood of mistakes higher. Greenphire was able to fulfill our request to allow a single entry to fill demographic information for both cards.

\section{Making patient payments}

Requests for payments to the stipend card are made from the local study site to the central site. Because few local sites had encrypted email systems, all requests are submitted by fax immediately after completion of a study visit. The high volume of faxes initially resulted in sites receiving a busy signal and faxes not being received. An e-fax system was purchased that allows receipt of up to 12 faxes simultaneously, thereby preventing delays in faxing. The e-fax system also allows us to file all faxes in an appropriate electronic study folder for backup.

To minimize data entry errors, the web-based ClinCard payment initiation site was developed with drop down menus that listed the number of the study visit and the specific payment for that visit. While additional ad hoc payments in other amounts can be made, having the drop down list of expected payments increases accuracy and efficiency in paying patients immediately following a confirmed study visit.

\section{Making Rx payments}

Use of the Rx card is initiated by a call from the pharmacy when a patient appears at the pharmacy to fill or refill a study prescription. The pharmacist or a pharmacy technician calls the toll free number on the Rx card. The call is answered by a BELT study staff member from the central work site (Olmsted Medical Center, MN). The ClinCard system is entered via the web. Using the patient's name, initials, or study ID number, the proper card listing is identified and the required amount loaded onto the card. While the only information that is required to make a payment is the amount of the requested payment, the BELT Rx system is set up to allow recording of the specific medications filled (name, dose and number of canisters) as well as the cost of each study medication. Hitting the "pay button" transfers the funds onto the card which can then be used as a credit card for payment and allows immediate dispensing of the medication(s).

\section{Customized reports}

The monthly summary reports from Greenphire designed for this study include information on total monthly and to-date medication expenditures, total monthly and to-date stipend expenditures, number of patients receiving medication funds, including any patients requesting more than one medication fill in a month, and a summary of other transactions, such as cards lost and replaced, or cards inactivated due to patient study completion or exit. Separate reports are provided for funds transferred to the Greenphire systems, charges for card uploads, and any other additional services provided. These reports support study accounting systems in place at the central site.

The ClinCard reports also help with assessing patient adherence. While the system cannot tell us who actually took the medications, it does provide information on medication refills. The comment box associated with each $\mathrm{Rx}$ card payment must be completed before funds can be uploaded and is used to record the exact medication given, including dose and cost (full, discounted, or copay cost) of each individual medication, given that in this study most patients are receiving two medications each month.

The fee to place funds on the cards is added automatically to the central site's invoices. Monthly statements are sent electronically and detail each amount placed on a card and all fees. The balance for each card system can be checked electronically on the Internet. Funds can be transferred electronically from the central coordinating center's bank at any time, and such transfers are only limited by the study site's finance office hours.

The web-based portal for the ClinCard system also has a feature for noting subjects who have completed the study. Using this feature, it is possible to block further uploads to the stipend or Rx cards after a patient completes the study. This is particularly helpful when a pharmacist calls for a refill on a weekend or at night when the study databases are not easily accessible. This feature assures that we do not provide any study medications beyond the period of the signed informed consent. 


\section{Results}

Over 1050 patient stipend and $\mathrm{Rx}$ cards have been distributed to patients for the BELT study. A total of 6741 interactions have been completed for the stipend card, totaling over \$250,000; an additional 8529 medication payments on the $\mathrm{Rx}$ card totaling over $\$ 680,000$ have been made. To date, the overall charges for ClinCard use have been slightly over $\$ 55,000$. Only two patients have refused the ClinCard system for visit payments, and both are paid by check from the central site, with funds arriving 10-14 days after the visits have been completed, rather than within 12 hours as happens with the ClinCard.

During the study period, several issues arose that required additional modifications to either the card system or to our methods of dealing with the cards. For the stipend card, patients found that the card could not easily be used in some businesses. For example, a few banks were unwilling to provide the patient with the cash on the card. This was not anticipated because the card instructions stated that transactions with banks should be convenient and free, as opposed to transactions at ATMs which were expected to result in user fees. ClinCard support contacted the banks to explain that this was a MasterCard. One bank did distribute funds, but the other continued to refuse because the card did not have the person's name on the front, instead saying only "valued customer" and the patient had no way of confirming that the card belonged to them. Using the card for gasoline purchases turned out to be problematic when patients tried to pay at the pump. When using the pay at the pump option, the parent gasoline company puts a hold on or sequesters funds on the card, often a $\$ 50$ hold and if the card did not have $\$ 50$ remaining, the card would be rejected for insufficient funds. Unfortunately, these sequestered funds would be held for a pending purchase and not returned to the card for a period of 3-7 days. Paying inside the station before or after completing the gas purchase solved this problem.

The credit card system does not work well for study screen failures, for which we paid the patient a small transportation fee. To provide the screen failure with a ClinCard for that payment, the central study would have to know the specific demographic information to assign a ClinCard; without the patient signing informed consent this was not possible. Patients who did not pass enrollment screening were instead sent cash. Gift cards with a predetermined cash value dispensed by the local study site would also solve this problem.

For patients who dropped out of the study or were exited, we were able to put a notice on their cards that they were exited but still allowed them to use any funds that remained on their stipend card. On a few occasions, patients found that a family member had used their card. This is possible since the card had no name listed, only "valuable customer". For these participants, the ability to develop a pin and use the card as a debit card was attractive as long as the patient did not forget his/her PIN, which could only be reset during weekday business hours.

A penalty for lack of activity on the card was charged by the company, although this was not anticipated. Information on the inactivity charges was included in the fine print of the card contract but was not noticed by the study staff when reviewing the provisions of card use. If the card had no activity for 6 months, $\$ 3$ was removed from the card every month until there was activity, with either additional funds added or funds used. This was only a problem for the stipend card because the funds placed on the Rx card were used immediately by the pharmacy. A federal law was passed in 2010 making nonuse fees illegal for credit and gift cards. Greenphire and MasterCard stated that the ClinCard penalty charges were not a use fee or a maintenance fee but a custodial fee which they interpret as being allowed. The company worked with us to help notify patients and reinstated fees charged before we were able to notify patients. Once we became aware of the fees, patients were sent notices warning them about nonuse charges.

The Rx card also met with some unanticipated barriers. Initially we had planned to place funds on the card prior to the visit to the pharmacy each month. However, this met with several problems. First, the patients did not always obtain medications from the same pharmacy. Secondly, the cost of medications varied from month to month, typically increasing in cost, and the pharmacist would have to call for insufficient funds. Finally, some patients realized that the $\mathrm{Rx}$ card had funds that could be used to make nonmedication purchases. The rate of this type of misuse of Rx funds was limited to less than $1 \%$ of the enrolled patients. Using ClinCard support, we were able to track those purchases made outside sites that had pharmacies, ie, restaurants and ATMs. We called each of those patients and suggested that they may have forgotten which card was for which type of purchase. We enhanced the initial training done by the local study coordinators regarding the Rx card to tell all patients that funds were only put onto the Rx card when the pharmacist called and the patient was on site to pick up the medication. A small number of patients genuinely tried to defraud the system by calling and claiming to be a pharmacist and asking for funds. These patients were recognized when they were 
unable to pronounce the medication name correctly, provide the dose, and usually quoted costs that were in rounded sums whereas all pharmacy charges included dollars and cents. Eight patients did obtain funds before we enhanced our system asking the pharmacist to provide dose and drug class information. At least five patients presented themselves to a pharmacy, allowed the pharmacist to call for the refill, and then when given back the card to run through the credit card machine left before swiping the card. These problems were discovered when the pharmacist called to say that the patient had not picked up the medication. We now request that the pharmacist swipe the Rx card and explain why we make this request on each pharmacy call.

A few pharmacists refused to call the number to inform us of the amount and medications to be purchased. Without this interaction, funds could not be placed on the Rx card and on a few occasions the patient paid for medications on their own or did not fill the prescriptions. In most cases, this was resolved by a call from one of the study investigators to the pharmacy explaining the details of the study. For frequently used pharmacies, a picture of the Rx card and a short note explaining the process were emailed to the pharmacy to be placed in the medication work area. Weekend "fill-in" pharmacists occasionally refused to call, telling patients that this was not part of their job. Patients could also call the Rx number when this happened and the pharmacist rarely refused to speak to the physician who answered the weekend and evening calls.

Study approval was problematic for only two of the 12 institutional review boards. These two institutional review boards were concerned about patient data being available to Greenphire and MasterCard. Greenphire was able to provide information regarding compliance with patient privacy regulations ${ }^{6}$ to assure the institutional review boards of their security systems and were reminded that informed consent documents explicitly outlined payment systems and required a patient's consent to use the system. The informed consent clearly stated that the card system for payment was like other credit cards that required specific demographic information such as name, address, and date of birth. Unlike other credit cards, no financial information was required and no health data were available because the study was always referred to as the BELT study and had no mention of lung disease and no lungs in the study logo (see Figures 1 and 2).

This study was designed to allow patients to obtain medications from their own personal pharmacy. Initial plans called for the Rx card to be uploaded with funds at a time convenient to the study team and that would be before a refill was anticipated. However, within a few weeks of study implementation, it became clear that the charge for the medication could vary widely from month to month and preloading the Rx card did not work. Patients were told they could go to their usual pharmacy and this meant that patients could and would refill medications during any hours the pharmacies were open, which almost always included nights and weekends. The ClinCard system actually facilitated this need by having a system that is web-based, and could therefore be accessed not only from a computer but also by an iPad or an iPhone. This allowed coverage of the study phone for pharmacy calls from any site that had an iPhone service available. The call coverage by iPhone still allowed access to the full patient history of medication use and costs, the patient's study ID number, and local site reference code. The ClinCard actually provided a feasible solution to this unanticipated study design issue.

\section{Discussion}

This clinical trial-specific credit card system has several features that have proven to be useful in providing patient stipends and medication payments in our real-world clinical trial. Use of the cards circumvented the need for local sites to keep cash or write checks to patients. Doing so would not only require extra time from the site for payments and tracking, but also open the local site to the possibility of financial compliance audits. The detailed reports from the monthly statements fulfilled the needs of the central site compliance audit and allowed all transactions to be electronic. Being a web-based system, access for payments could be made via a personal device that allows Internet access while maintaining security through use of passwords that were changed regularly. The system also allows tracking of nonfiscal study compliance, such as patient visits and medication dispensing. The reports have met the needs of the outside auditors for government funding review and facilitate our ability to address pharmacy, patient, and investigator queries quickly regarding payments and medications obtained.

Some of the sites were concerned that patients would not be able to use the system. To date, we have had very few problems, other than patients forgetting that they had already used their funds, but the ability to tell them when and the amount of expenditures from the stipend card has addressed these concerns. The sites are very pleased to not have to keep any cash on hand or to have to keep the records required for confirmation of dispensing funds.

The ClinCard system works well for our complex study and multiple types of payments to patients (medications and 
stipends for visits). With limited extra support through phone calls, patients have learned to use the system, even patients who the coordinators thought might have trouble understanding the system. Most pharmacy staff members are willing to call and report the medications to be obtained and the charge for those medications. Some pharmacists are even kind enough to call and report medications picked up when there are no copays and therefore no upload to the Rx card is required. One or two substitute pharmacists have been unwilling to call, so patients have paid for medications and been reimbursed or have simply gone to another pharmacy. We have asked all patients not to go through the drive-through pharmacy windows, because the time for fund transfers to the card may be up to 10-15 minutes at busy times of the day (busy for both the staff and the card company) and this delays the drive-through lines.

All of the issues identified were easier to address due to the responsiveness of the ClinCard staff. Even after work hours, a helpline number was available and responses were usually within a few hours. For issues of payment confusion requiring reconciliation or patients who apparently forgot they had used their cards, ClinCard was able to identify expenditure dates, times, and amounts, helping patients remember that they had spent the funds. After being able to provide this detailed information, we seldom received a second call from any patient regarding concerns about "missing" funds.

The study design did require a person to be available for the study phone during all hours that pharmacies were open. While this was not anticipated, it is the result of the study design not the ClinCard system. The ability of the system to be accessed by personal mobile devices actually facilitated this night and weekend call requirement.

\section{Conclusion}

The ClinCard and other similar systems facilitate patient payments for study visits and variable real-time payments for medications obtained from community pharmacies. The system provides tracking to help assess completed visits, medication adherence, and total payments to patients for tax purposes. The system requires Internet access, but payments can be loaded from mobile devices, including an iPad or iPhone. Costs for the system are modest and are offset by eliminating the need for separate accounting records for patient payments, medication payments, and reports that facilitate tracking of patient adherence with study visits and obtaining study medications. The system is flexible and has customizable features for patient visit reminders (email or text messaging), limits on amounts placed on cards at any one time, ability to void immediately any transaction that was made in error, and electronic and printable summaries of all card transactions by the study team. For widely distributed study sites and community-based practices unable or unwilling to deal with onsite payments, this system has been used with ease and confidence. Such systems are likely to replace the need for coordinating centers to develop duplicate monitoring and payment systems.

\section{Disclosure}

No funding was received from the makers of the system described. While the specific system used is named, the authors did not notify the business prior to preparation of the manuscript. However, the business was allowed the opportunity to review the manuscript for potential inaccuracies prior to submission for publication. The authors declare that there are no conflicts of interest in this work.

\section{References}

1. Moral Science. Protecting participants in human subjects research. Presidential Commission for the Study of Bioethical Issues. Washington, DC. Dec 2001. Available from: http://www.bioethics.gov. http://bioethics.gov/cms/sites/default/files/Moral\%20Science $\% 20$ (Updated $\% 20$ 2012)_0.pdf. Accessed November 1, 2012.

2. Menikoff J. Just compensation: paying research subjects relative to the risk they bear. Am J Bioeth. 2001;1:56-58.

3. Reiser SJ. Research compensation and the monetarization of medicine. JAMA. 2005;293:613-614.

4. Anderson JA, Weijer C. The research subject as wage earner. Theor Med Bioeth. 2002;23:359-376.

5. Juniper EF, Bousquet J, Abetz L, Bateman ED. Identifying 'wellcontrolled' and 'not well-controlled' asthma using the Asthma Control Questionnaire. Respir Med. 2006;100:616-621.

6. Greenphire.com [homepage on the Internet]. King of Prussia, PA: Greenphire. Available from: http://www.greenphire.com/. Accessed September 24, 2012.

7. ClearTrial.com [homepage on the Internet]. Redwood Shores, CA: Oracle Corporation. Available from: http://www.cleartrial.com. Accessed November 2, 2012.

8. BioClinica.com [homepage on the Internet]. Newton, PA: Bioclinica. Available from: http://www.bioclinica.com/clinical-payment-manager. Accessed September 18, 2012.

9. Appliedclinicaltrialsonline.com [homepage on the Internet]. Iselin, NJ: Advanstar. Available from: http://www.appliedclinicaltrialsonline. com/appliedclinicaltrials/article/articleDetail.jsp?id=742593. Accessed September 24, 2012.

10. Centerwatch.com [homepage on the internet]. Boston, MA: CenterWatch. Available from: http:/www.centerwatch.com/newsonline/company/Greenphire. Accessed September 24, 2012. 
Open Access Journal of Clinical Trials

Dovepress

\section{Publish your work in this journal}

The Open Access Journal of Clinical Trials is an international, peerreviewed, open access journal publishing original research, reports, editorials, reviews and commentaries on all aspects of clinical tria design, management, legal, ethical and regulatory issues, case record form design, data collection, quality assurance and data auditing methodologies. The manuscript management system is completely online and includes a very quick and fair peer-review system, which is all easy to use. Visit http://www.dovepress.com/testimonials.php to read real quotes from published authors.

Submit your manuscript here: http://www.dovepress.com/open-access-journal-of-clinical-trials-journal 